Article

\title{
Zinc Differentially Modulates the Assembly of Soluble and Polymerized Vimentin
}

\author{
Andreia Mónico, Silvia Zorrilla, Germán Rivas and Dolores Pérez-Sala * \\ Department of Structural and Chemical Biology, Centro de Investigaciones Biológicas Margarita Salas, \\ Ramiro de Maeztu, 9, 28040 Madrid, Spain; andreiamonico89@gmail.com (A.M.); silvia@cib.csic.es (S.Z.); \\ grivas@cib.csic.es (G.R.) \\ * Correspondence: dperezsala@cib.csic.es
}

Received: 7 March 2020; Accepted: 30 March 2020; Published: 31 March 2020

\begin{abstract}
The intermediate filament protein vimentin constitutes a critical sensor for electrophilic and oxidative stress. We previously showed that vimentin interacts with zinc, which affects its assembly and redox sensing. Here, we used vimentin wt and C328S, an oxidation-resistant mutant showing improved $\mathrm{NaCl}$-induced polymerization, to assess the impact of zinc on soluble and polymerized vimentin by light scattering and electron microscopy. Zinc acts as a switch, reversibly inducing the formation of vimentin oligomeric species. High zinc concentrations elicit optically-detectable vimentin structures with a characteristic morphology depending on the support. These effects also occur in vimentin C328S, but are not mimicked by magnesium. Treatment of vimentin with micromolar $\mathrm{ZnCl}_{2}$ induces fibril-like particles that do not assemble into filaments, but form aggregates upon subsequent addition of $\mathrm{NaCl}$. In contrast, when added to $\mathrm{NaCl}$-polymerized vimentin, zinc increases the diameter or induces lateral association of vimentin wt filaments. Remarkably, these effects are absent or attenuated in vimentin C328S filaments. Therefore, the zinc-vimentin interaction depends on the chemical environment and on the assembly state of the protein, leading to atypical polymerization of soluble vimentin, likely through electrostatic interactions, or to broadening and lateral association of preformed filaments through mechanisms requiring the cysteine residue. Thus, the impact of zinc on vimentin assembly and redox regulation is envisaged.
\end{abstract}

Keywords: vimentin; zinc; cysteine; redox sensing; intermediate filaments; cysteine mutant; filament bundling; filament width; divalent cations; magnesium

\section{Introduction}

Intermediate filaments play a fundamental role in the integration of cytoskeletal functions, and consequently in cell dynamics and behavior. The type III intermediate filament protein vimentin constitutes a key cytoskeletal element of mesenchymal cells. Vimentin plays key roles in essential cell functions such as division and migration and contributes to cellular structural support and plasticity and organelle positioning. In addition, recent reports have unveiled its complex interplay with other cytoskeletal systems [1,2] and its involvement in redox sensing [3,4], regulation of gene expression, [5] or protection of the nucleus and DNA from damage [6]. Moreover, biophysical techniques are providing high resolution information on the mechanics and dynamic performance of the vimentin network, with a clear impact on the physical properties of cells [6,7]. In pathophysiology, vimentin is a key marker and agent of epithelial mesenchymal transition and tumor malignancy $[2,8-10]$ and is involved in bacterial and viral infections [11] and autoimmune diseases [12].

Vimentin's structure is comprised of intrinsically disordered N-terminal (head) and C-terminal (tail) domains and a central rod segment of a coiled-coil structure. In vitro, vimentin monomers spontaneously form dimers and tetramers when purified from solutions containing chaotropic agents 
into low ionic strength buffers. In turn, these oligomers rapidly form filaments upon raising the ionic strength. Numerous excellent works have delineated the process of vimentin filament assembly starting from the association of parallel dimers into staggered antiparallel tetramers, and of these into "unit length filaments" (ULF), several of which engage end-to-end to form filaments that subsequently undergo a radial compaction process to yield mature filaments [13-15].

In cells, vimentin is subjected to an exquisite regulation, both by transcriptional and posttranscriptional mechanisms relying on a plethora of posttranslational modifications, including phosphorylation, glycosylation, SUMOylation, as well as non-enzymatic processes, like oxidative modifications $[4,16]$. In particular, modifications of the vimentin single cysteine residue, C328 in human vimentin, play a key role in filament assembly in vitro and network remodeling in cells $[3,4,17]$. Oxidative modifications of C328 disrupt in vitro filament formation in a manner dependent on the structure of the modifying moiety [4]. Moreover, C328 appears to occupy a key position in assembled filaments in such a way that modifications or substitutions of this residue by other amino acids affect vimentin performance in cellular functions. Thus, C328S vimentin is less efficient than the wild-type $(\mathrm{wt})$ protein at forming extended networks and supporting organelle positioning in cells [3]. Moreover, cysteine-deficient vimentin displays an attenuated response against several types of stress [3,4]. Although knowledge of the regulation of vimentin is rapidly expanding, there are still several intriguing aspects like its potential interaction with regulatory or associated proteins and the mechanisms controlling assembly in the cellular context.

Given its amino acid composition, vimentin is known to behave as a polyelectrolyte and to interact with cations through charge-mediated interactions [18]. The interaction of vimentin with divalent cations, mainly magnesium and calcium, has long been known and characterized in detail in vitro [19-21]. Both magnesium and calcium have been shown to induce vimentin polymerization on their own when used at millimolar concentrations [20,22,23] and to increase the stiffness of vimentin networks, thereby acting as crosslinkers. Indeed, divalent cations were found to interact with the tail domain of vimentin, in particular with the last 11 amino acids, inducing its crosslinking [19]. In particular, $\mathrm{Mg}^{2+}$ ions appear to accumulate in the tail segments due to the abundance of negatively charged amino acids in this domain [24]. In microfluidic drops, concentrations of magnesium in the millimolar range augmented the compaction of preassembled (KCl-induced) vimentin filament networks until they became completely aggregated $[25,26]$. In cells, the role of divalent cations on vimentin structure and function can be highly complex. Besides a direct interaction with the protein, divalent cations could act on vimentin dynamics through multiple mechanisms, including regulation of proteases or kinases in the case of calcium [27-29], binding to ATP for kinase reactions or facilitating GTP binding to GTPases in the case of magnesium.

Zinc is the transition metal that most frequently acts as a cofactor of proteins [30]. Zinc interaction with proteins may play structural and catalytic roles or serve for its storage and transport in cells. Importantly, zinc availability has clear pathophysiological implications in diseases related to protein aggregation, oxidative stress, and immune function [3]. Zinc can interact with proteins with beneficial or deleterious consequences. Thus, zinc can provide interactions promoting protein stability, but also facilitates the formation of pathogenic aggregates, such as $A \beta$ amyloid precipitates or Tau fibrils [31,32]. On the other hand, zinc can induce cellular effects by modulating the activity of transcription factors [33] and phosphatases [34]. Moreover, zinc can influence protein and cellular redox status due to its potential pro-antioxidant or pro-oxidant roles $[35,36]$. Therefore, the interactions between zinc and vimentin in vitro and in cells can be complex, and their delineation requires careful assessment.

The interaction of intermediate filament proteins with zinc was first noted for keratins, in which low millimolar zinc induced protein aggregation, elicited the reversible association of two to four keratin filaments, and promoted the formation of macrofilaments $[37,38]$. While studying the importance of the single cysteine residue of vimentin, we obtained evidence indicating that zinc could interact with vimentin in vitro, protecting this residue from alkylation [3]. Moreover, zinc promoted vimentin polymerization into insoluble structures per se. In cells, we observed that zinc availability 
contributed to network robustness and protection from oxidants [3]. Our observations suggested a supra-stoichiometric interaction of vimentin with zinc with preference over other divalent cations such as $\mathrm{Mg}^{2+}$. This led us to propose a role for the zinc-vimentin interaction in both vimentin and zinc homeostasis $[39,40]$.

Nevertheless, the structural basis of the vimentin-zinc interaction is not known. Here, we studied the effect of zinc on vimentin assembly in vitro. Our results indicated that low micromolar zinc induced a differential modulation of the assembly in soluble vimentin and preformed filaments, which could have implications in physiological or pathophysiological processes.

\section{Results}

\subsection{EDTA-Free Vimentin Reveals Differential Polymerization Features Derived from the Presence of the Cysteine Residue}

Most protocols for purification and refolding of vimentin employ millimolar concentrations of EDTA as the stabilizing agent and metalloprotease inhibitor. Therefore, in order to study the effect of micromolar zinc on vimentin assembly, we employed a procedure for efficient removal of EDTA from vimentin preparations [41]. This procedure, which involves ultrafiltration and dialysis, yielded vimentin as a single band in SDS gels, even under non-reducing conditions, indicating that the protein is recovered in its reduced form (Figure 1A). This is important since we have previously shown that vimentin oxidation can hamper filament formation [4]. The functionality of the resulting protein preparation was validated by polymerization assays. Both vimentin wt and its C328S mutant were recovered in the pellet fraction from ultracentrifugation after addition of $150 \mathrm{mM} \mathrm{NaCl}$, indicative of polymerization (Figure 1B). Next, the morphology of the structures present and the kinetics of polymerization were assessed by electron microscopy (EM). In the absence of $\mathrm{NaCl}$, vimentin preparations appeared as homogeneous lattices of rod-like structures of $2-5 \mathrm{~nm}$ in width, generally shorter than $50 \mathrm{~nm}$ (Figure 1C), which likely correspond to the soluble oligomeric vimentin forms previously characterized [42]. Upon addition of $\mathrm{NaCl}$, the formation of typical vimentin filaments was observed from the first time-point assessed, i.e., $5 \mathrm{~min}$, at which, filament width was approximately $14 \mathrm{~nm}$ (Figure 1D). At later polymerization times, filaments narrowed gradually, in accordance with the radial compaction phenomenon (quantitated in Figure 1E). Interestingly, vimentin wt filaments were consistently wider than vimentin C328S filaments, reaching 12.3 and $11.8 \mathrm{~nm}$ in diameter, respectively (Figure 1E). In turn, vimentin C328S filaments were significantly longer than wt at early polymerization times (Figure 1F), suggesting a faster elongation and highlighting a role of the residue at position 328 in the assembly process. In summary, these virtually EDTA-free vimentin preparations were considered functionally appropriate to study the effect of zinc on vimentin oligomerization. Moreover, they allowed the observation of differences in the polymerization behavior of wt and C328S vimentin.

\subsection{Micromolar Zinc Reversibly induces Vimentin Oligomerization}

The effect of zinc on vimentin polymerization was studied first by ultracentrifugation assays (Figure 2A). Analysis of the supernatant and pellet fractions containing soluble and insoluble-polymerized vimentin, respectively, showed that micromolar $\mathrm{ZnCl}_{2}$ induced the polymerization of both vimentin wt and C328S, in such a way that in the presence of $250 \mu \mathrm{M} \mathrm{ZnCl}$, most of the vimentin was in the pellet. Although informative, centrifugation assays require long processing times. In order to study the effect of zinc on vimentin polymerization in more detail, we employed turbidity assays, which allow monitoring zinc-induced changes in real time (Figure 2B). These showed a steady increase in turbidity with increasing zinc concentrations for both vimentin wt and C328S, which reflects the formation of larger vimentin species already at concentrations of $\mathrm{ZnCl}_{2}$ in the micromolar range. An increase in turbidity did not saturate at the highest concentration used $(300 \mu \mathrm{M})$. Nevertheless, higher concentrations or longer turbidity measurements were not considered due to decreases in absorbance compatible with sedimentation of large protein assemblies. 
A

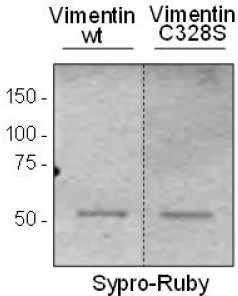

Non-reducing

conditions

C

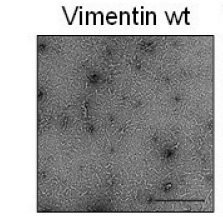

B
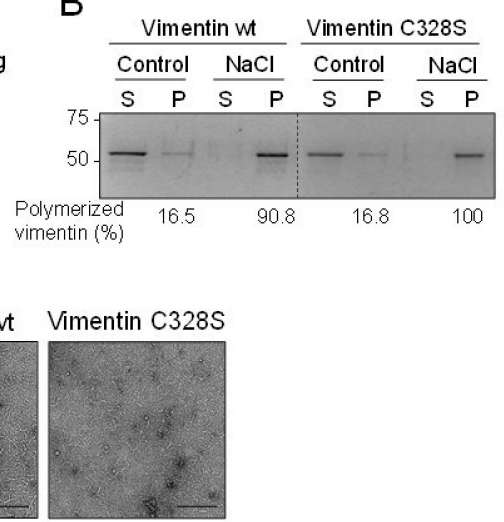

D
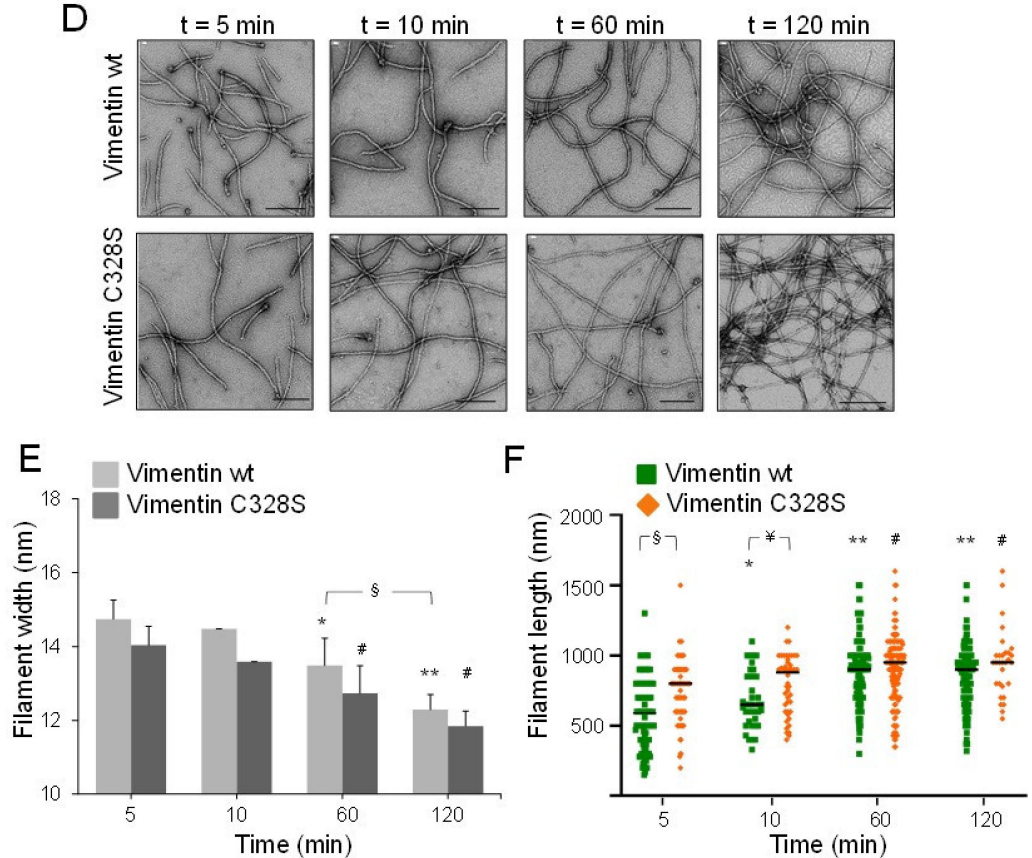

Figure 1. Polymerization of vimentin wt and C328S. (A) Vimentin purified and subjected to EDTA removal was analyzed by gel electrophoresis under non-reducing conditions. Total protein was stained with Sypro-Ruby. The position of molecular weight markers is indicated on the left. (B) Vimentin wt or C328S in $5 \mathrm{mM}$ PIPES-Na pH 7.0, $0.2 \mathrm{mM}$ DTT was incubated with $150 \mathrm{mM} \mathrm{NaCl}$ for $15 \mathrm{~min}$ at $37^{\circ} \mathrm{C}$ and polymerization assessed by ultracentrifugation. Supernatant $(\mathrm{S})$ and pellet $(\mathrm{P})$ fractions, containing soluble and polymerized vimentin, respectively, were analyzed by SDS-PAGE and Sypro-Ruby staining. The amount of vimentin in each fraction was estimated by image scanning, and the percentage of polymerized vimentin is shown below the gel. Results are representative of five assays. (C) Soluble vimentin wt and C328S preparations were analyzed by electron microscopy (EM). Images are representative of four and 10 preparations of vimentin wt and C328S, respectively. (D) Vimentin assembly was induced by the addition of $150 \mathrm{mM} \mathrm{NaCl}$ at $37^{\circ} \mathrm{C}$, and filament formation at the indicated times was monitored by EM. Images are representative of at least three assays. Images were analyzed with FIJI to determine filament width and length under the different conditions for both vimentin preparations, and the results are shown in $(\mathbf{E})$ and $(\mathbf{F})$, respectively. Results are average values \pm SEM of 50 to 200 filaments per condition. In (E), ${ }^{*} p<0.05$, and ${ }^{* *} p<0.001$ vs. vimentin wt at $5 \mathrm{~min}, \# p<0.05$ vs. vimentin C328S at $5 \mathrm{~min}, \S p<0.05$, as indicated. In $(\mathbf{F}),{ }^{*} p<0.05$, and ${ }^{* *} p<0.0001$ vs. vimentin wt at $5 \mathrm{~min}, \# p<0.05$ vs. vimentin C328S at $5 \mathrm{~min}, \S p<0.0001$, $¥ p<0.05$, as indicated, by the unpaired Student's $t$-test. Scale bars, $200 \mathrm{~nm}$. 
A
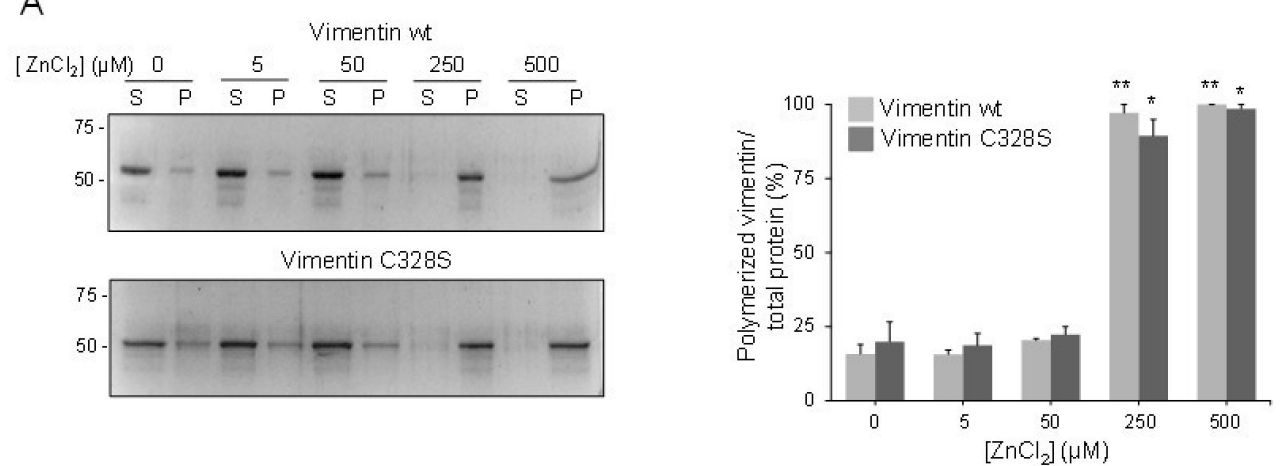

B

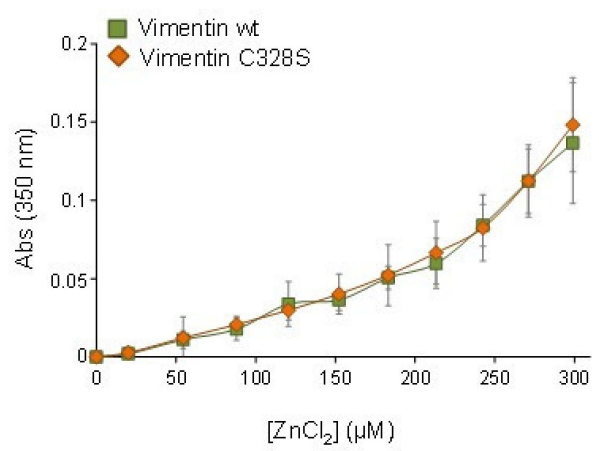

C
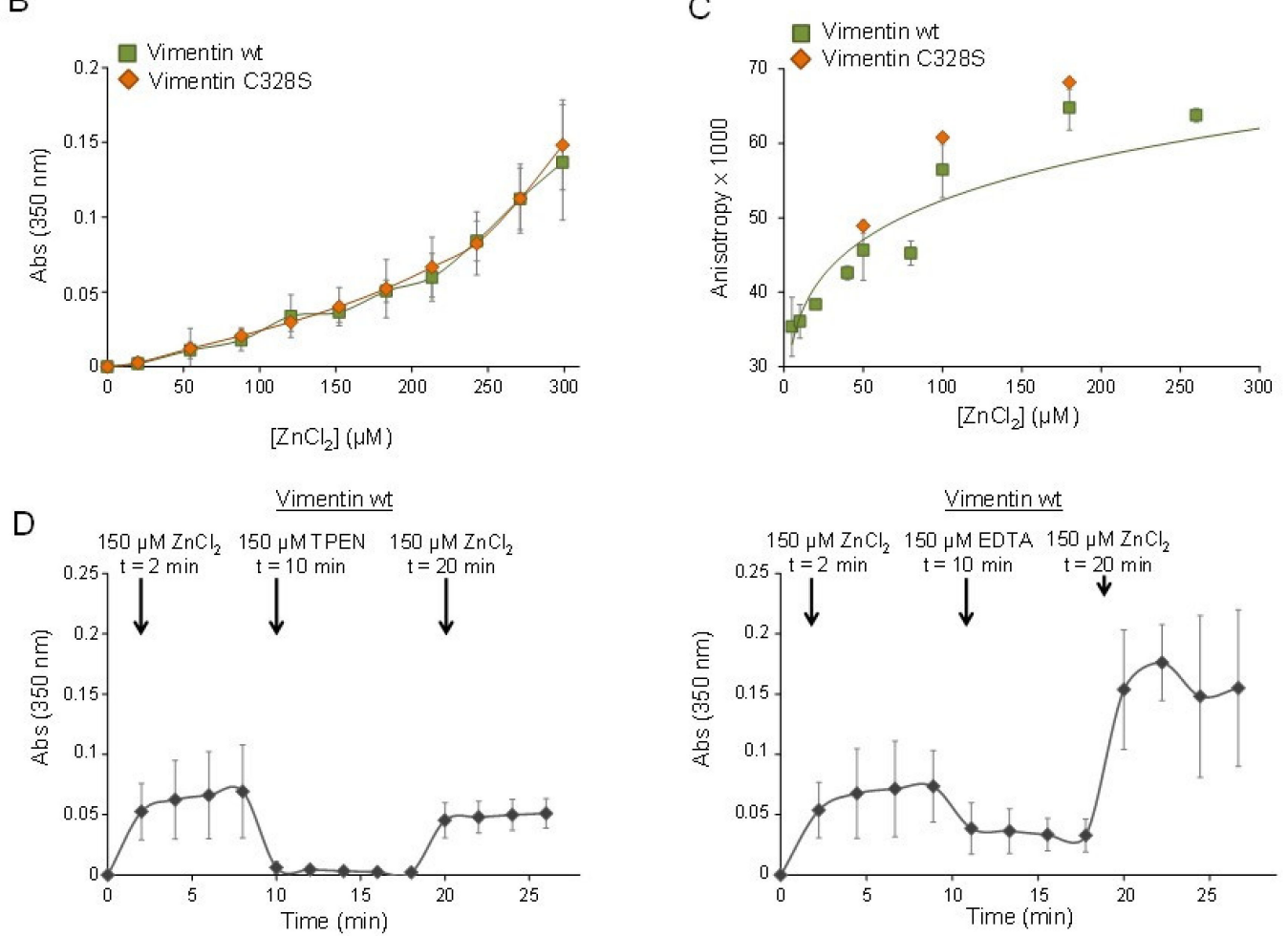

Figure 2. Effect of micromolar zinc concentrations on vimentin oligomerization assessed by centrifugation and turbidity assays. (A) Vimentin wt or C328S was incubated with the indicated concentrations of $\mathrm{ZnCl}_{2}$ for $1 \mathrm{~h}$ at r.t., and the formation of insoluble oligomers was assessed by ultracentrifugation as above. Gels shown are representative of three assays. The proportion of polymerized vimentin, i.e., present in the pellet fraction, was estimated by image scanning, and results corresponding to the mean \pm SEM of three assays are depicted in the graphs. ${ }^{* *} p<0.005$, and ${ }^{*} p<0.05$ vs. the corresponding construct in the absence of zinc. (B) Vimentin wt or C328S was incubated with increasing concentrations of $\mathrm{ZnCl}_{2}$, and the turbidity of the solution, assessed as the absorbance at $350 \mathrm{~nm}$ at r.t., was measured immediately. Results are the mean values $\pm \mathrm{SD}$ of at least three experiments. (C) Mixtures containing $1 \mu \mathrm{M}$ unlabeled vimentin and $50 \mathrm{nM}$ Alexa 488-labeled vimentin were incubated with increasing concentrations of $\mathrm{ZnCl}_{2}$, and fluorescence anisotropy was monitored at $26^{\circ} \mathrm{C}$. Results are the mean $\pm \mathrm{SD}$ of eight (vimentin wt) or two replicates (vimentin C328S). (D) Reversibility of zinc-induced vimentin aggregation upon addition of TPEN (left) or EDTA (right). Vimentin was sequentially incubated with $\mathrm{ZnCl}_{2}$, TPEN, or EDTA and, then, $\mathrm{ZnCl}_{2}$ again at r.t., at the concentrations and time-points indicated. Results are average values from three independent experiments. Lines in the graphs are only meant to guide the eye. 
Next, fluorescence anisotropy was employed to confirm the formation of oligomeric assemblies in the presence of micromolar zinc. Mixtures of unlabeled vimentin wt or C328S and the corresponding construct labeled with Alexa 488, as a tracer, were incubated with increasing concentrations of $\mathrm{ZnCl}_{2}$, and fluorescence anisotropy was measured (Figure 2C). A concentration-dependent increase in fluorescence anisotropy, compatible with the formation of larger protein species, was clearly observed with concentrations of $\mathrm{ZnCl}_{2}$ below $50 \mu \mathrm{M}$ and corroborated the ability of micromolar zinc to induce vimentin oligomerization. Similar to the turbidity measurements, no significant differences in zinc-induced aggregation between vimentin wt and C $328 \mathrm{~S}$ were observed. Turbidity measurements were used to explore the dynamic nature of zinc-induced vimentin oligomerization. As shown in Figure $2 \mathrm{D}$, incubation of vimentin with $150 \mu \mathrm{M} \mathrm{ZnCl}_{2}$ elicited a nearly immediate increase in turbidity that was stable during the time of observation. The addition of an equivalent concentration of the metal chelators, TPEN or EDTA, which form a 1:1 complex with zinc, immediately caused an abrupt decrease in turbidity, compatible with disassembly of zinc-induced oligomers. This decrease was more marked in the case of TPEN, which reduced turbidity down to basal levels. This could be related to the higher affinity of TPEN for zinc, compared to EDTA $\left(\mathrm{K}_{\mathrm{d}}\right.$ of $6.4 \times 10^{-16} \mathrm{M}$ and $6 \times 10^{-14} \mathrm{M}$, for TPEN and EDTA, respectively) [43]. Interestingly, subsequent re-addition of $150 \mu \mathrm{M} \mathrm{ZnCl}_{2}$, final concentration, rapidly increased turbidity again up to levels similar to those attained after the initial addition of $\mathrm{ZnCl}_{2}$ in the presence of TPEN, but apparently higher in the presence of EDTA. This may reflect a lower chelating power of EDTA or additional effects on vimentin assembly [42] that call for caution in the use of this chelator. Nevertheless, these observations clearly show that the effect of zinc on vimentin oligomerization is reversible and suggest that micromolar zinc can act as a switch eliciting the formation of vimentin oligomeric species. Moreover, these results parallel observations in cells showing the cyclic disassembly and re-assembly of GFP-vimentin constructs upon addition and withdrawal of TPEN [3].

Importantly, the effect of zinc could not be mimicked by similar concentrations of another divalent cation, namely, $\mathrm{Mg}^{2+}$. As shown in Figure 3A, adding $\mathrm{MgCl}_{2}$ up to $500 \mu \mathrm{M}$ did not increase the proportion of insoluble vimentin for any of the constructs. Turbidity assays demonstrated that millimolar concentrations of $\mathrm{MgCl}_{2}$ were required to elicit increases in absorbance similar to those induced by micromolar zinc (Figure 3B). For instance, a comparable increment in turbidity was observed with $150 \mu \mathrm{M} \mathrm{ZnCl}_{2}$ and $8 \mathrm{mM} \mathrm{MgCl}_{2}$, that is a 50 -fold higher concentration of magnesium. These observations indicated that the mode of binding of the two divalent cations to the protein should be different, which could potentially result in distinct structural consequences.

Finally, we assessed the impact of several $\mathrm{NaCl}$ concentrations on vimentin turbidity and found that millimolar $\mathrm{NaCl}$ only marginally increased turbidity under the conditions of this assay (Figure 3C).

\section{3. the Morphology of Zinc-Induced Vimentin Assemblies Depends on the Support}

Turbidity assays provide a quantitative measurement of vimentin oligomerization. However, they do not provide information on the morphology of the assemblies formed. Therefore, we employed several microscopy techniques to assess this point (Figure 4). When a solution of vimentin was incubated with increasing concentrations of $\mathrm{ZnCl}_{2}$ in a flat-bottomed plastic microplate, vimentin formed assemblies with a garland-like appearance that were detectable after 10 to $20 \mathrm{~min}$ of incubation with $200 \mu \mathrm{M} \mathrm{ZnCl}_{2}$ and became more abundant with higher zinc concentrations (Figure 4A).

The formation of assemblies in a confined environment was also monitored by using a mixture of unlabeled vimentin wt and vimentin wt labeled with Alexa 488 (4:1), encapsulated in picoliter-sized aqueous droplets stabilized by lipids in an oil phase, and observed by fluorescence microscopy (Figure 4B). In the absence of zinc, vimentin was homogenously dispersed inside the droplets. Low concentrations of $\mathrm{ZnCl}_{2}$, e.g., $20 \mu \mathrm{M}$, did not alter this pattern detectably. However, when encapsulated in the presence of 100 to $200 \mu \mathrm{M}$ concentrations of $\mathrm{ZnCl}_{2}$, polymorphic aggregates and occasional filamentous structures could be observed, causing an obvious redistribution of fluorescence (Figure 4B). 
A
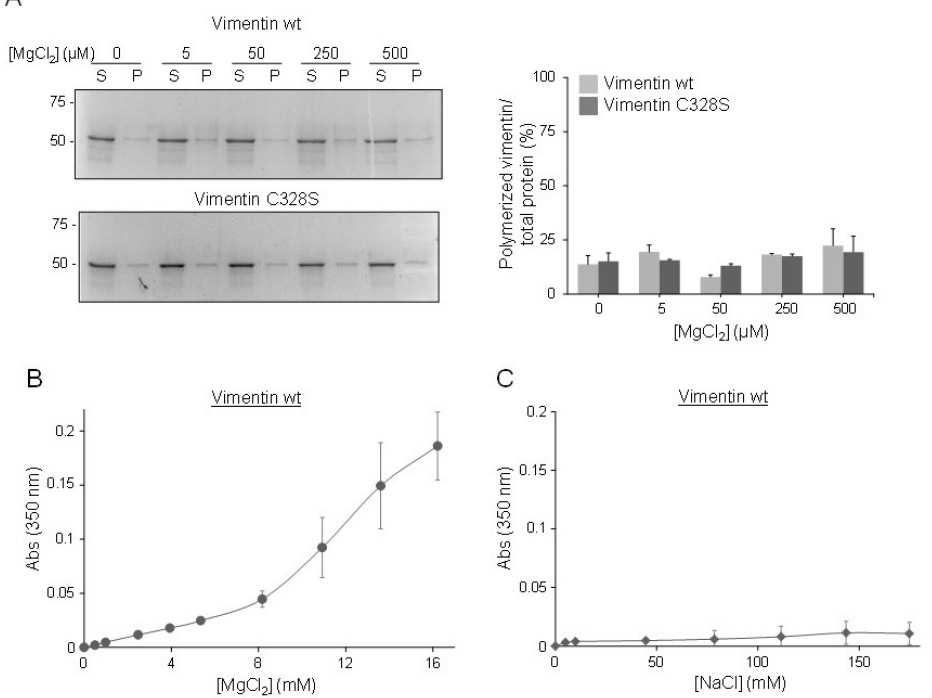

Figure 3. Effect of $\mathrm{MgCl}_{2}$ or $\mathrm{NaCl}$ on vimentin polymerization. (A) Vimentin wt or C328S in $5 \mathrm{mM}$ PIPES-Na pH 7.0, 0.2 mM DTT, was incubated with the indicated concentrations of $\mathrm{MgCl}_{2}$ for $1 \mathrm{~h}$ at r.t., and polymerization was assessed by ultracentrifugation as above. Supernatant $(\mathrm{S})$ and pellet $(\mathrm{P})$ fractions, containing soluble and polymerized vimentin, respectively, were analyzed by SDS-PAGE and staining with Sypro-Ruby. The percentage of polymerized vimentin for every condition is shown in the graph as average values \pm SEM from two assays. (B,C) Vimentin wt was incubated with increasing concentrations of $\mathrm{MgCl}_{2}$ (B) or $\mathrm{NaCl}(\mathbf{C})$ and turbidity measured at $350 \mathrm{~nm}$ at r.t. Results are the mean $\pm \mathrm{SD}$ of three $(\mathbf{B})$ or two $(\mathbf{C})$ assays.

Consistent with our previous observations, when mixed with $\mathrm{ZnCl}_{2}$ on glass coverslips, vimentin immediately formed aggregates and arrays or fibers, the robustness of which increased with zinc concentration (Figure 4C). Moreover, these fibers could be stained with zinquin, a fluorescent probe that detects both free and protein-bound zinc. Zinc also induced the formation of fibers of vimentin C328S (Figure 4C). Conversely, incubation of vimentin on glass with equivalent concentrations of $\mathrm{MgCl}_{2}$ elicited some amorphous aggregates, but no fibers were detectable by optical microscopy, indicating that the zinc-vimentin interaction possesses specific features (Figure 4C).

Therefore, the morphology of the zinc-induced vimentin assemblies formed appears to depend on the support used and the environmental conditions.

\subsection{Assessment of Zinc-Induced Vimentin Structures by Electron Microscopy}

Optical microscopy provides information on the morphology of the larger assemblies of vimentin. In order to assess the effects of zinc on smaller assemblies, we employed EM (Figure 5). As described above, vimentin in hypotonic buffer appeared mainly as weakly-defined small rod-like structures of $2-5 \mathrm{~nm}$ in width and $40-50 \mathrm{~nm}$ in apparent length (Figure $5 \mathrm{~A}$ ). Upon incubation with $10 \mu \mathrm{M} \mathrm{ZnCl}_{2}$, vimentin formed more easily distinguishable assemblies, consisting of rod-shaped particles of $9.70 \pm$ $0.03 \mathrm{~nm}$ and $9.43 \pm 0.03 \mathrm{~nm}$ in width, in vimentin wt and C328S, respectively, which coexisted with some non-assembled material and occasional larger aggregates. Moreover, $100 \mu \mathrm{M} \mathrm{ZnCl}_{2}$ further increased the diameter of vimentin structures up to $10.6-11.5 \mathrm{~nm}$, as depicted in Figure 5B. Remarkably, the length of zinc-induced structures did not suffer a noticeable variation with increasing zinc concentrations over the time of the experiment $(1 \mathrm{~h}$ ) (Figure $5 \mathrm{C}$ ). Thus, these observations indicated that zinc promoted lateral association of vimentin oligomers into fibrils. Nevertheless, on average, these structures did not reach the dimensions typical of ULF, which display a characteristic length of approximately $60-65 \mathrm{~nm}$ and $16-20 \mathrm{~nm}$ in width $[13,44]$. Moreover, elongation or end-to end annealing of these fibrils did not appear to take place in a significant proportion, since the structures observed did not reach the length corresponding to two typical ULF during the time frame of the experiment (length was always below $100 \mathrm{~nm}$ ). 
A

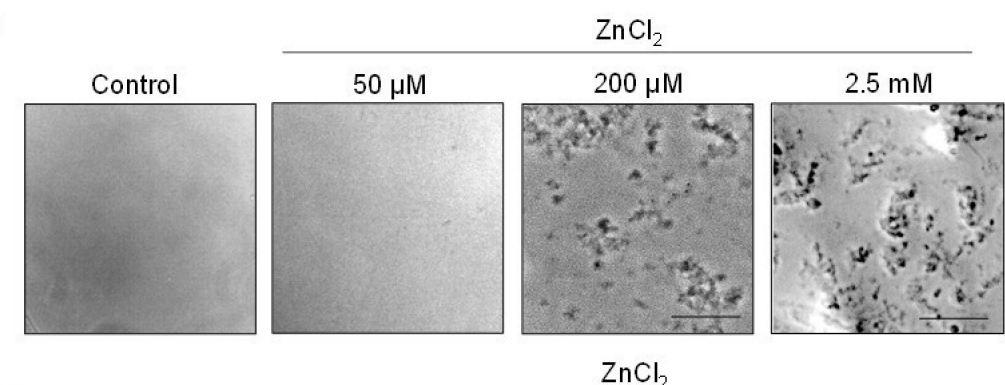

B
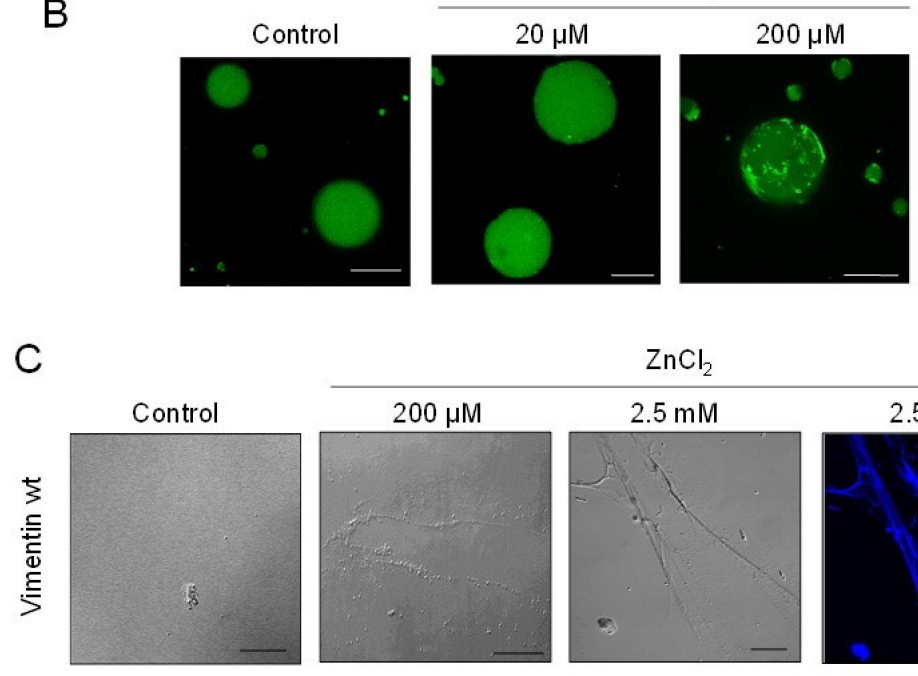

$\mathrm{ZnCl}_{2}$
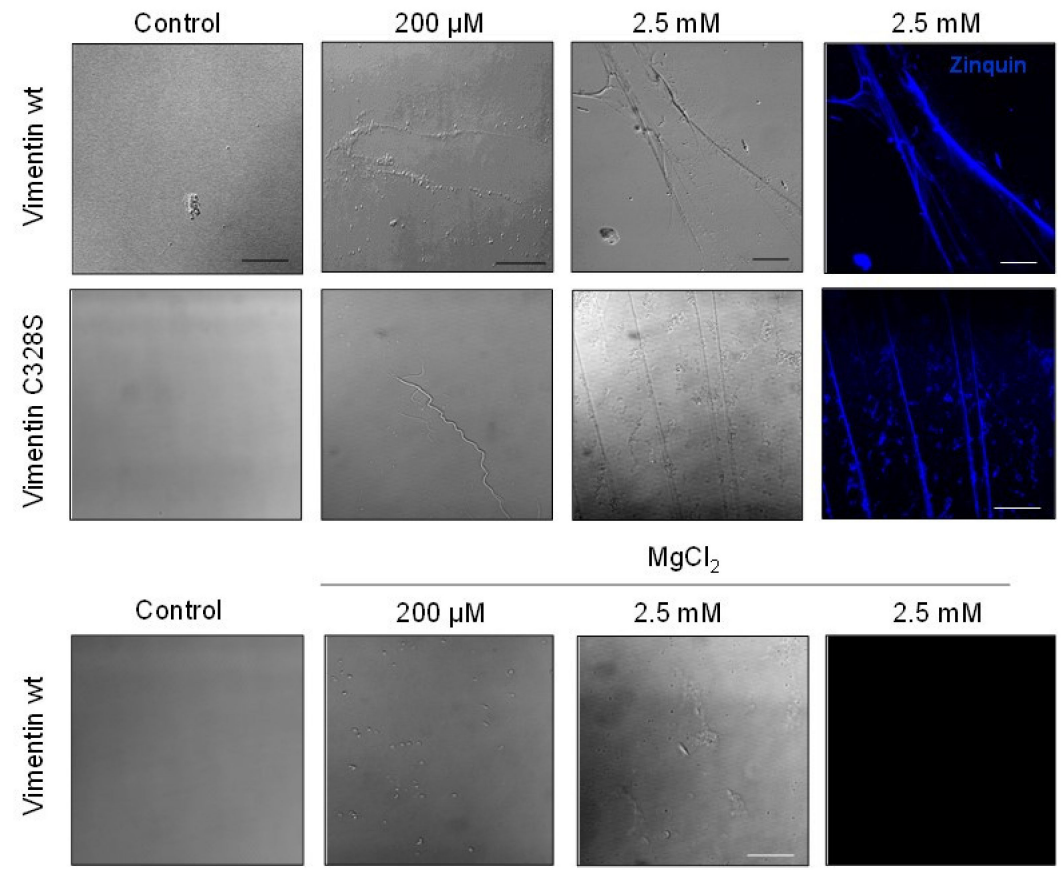

$\mathrm{MgCl}_{2}$

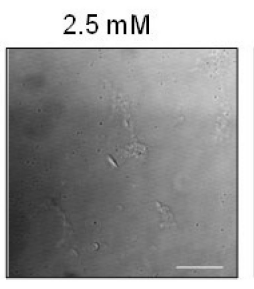

$2.5 \mathrm{mM}$

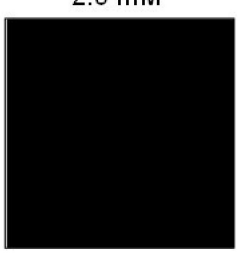

Figure 4. Observation of zinc-induced vimentin structures by optical microscopy. (A) Vimentin wt in 5 mM PIPES-Na pH 7.0, 0.2 mM DTT, was incubated with the indicated concentrations of $\mathrm{ZnCl}_{2}$ in a plastic microwell plate. Mixtures were observed after $20 \mathrm{~min}$ incubation at r.t. Images are representative of three assays. Scale bar, $20 \mu \mathrm{m}$. (B) Vimentin mixtures containing unlabeled and Alexa 488-labeled vimentin ( 4 and $1 \mu \mathrm{M}$, respectively) were encapsulated in microdroplets in the presence of the indicated concentrations of $\mathrm{ZnCl}_{2}$, at r.t., and observed by confocal fluorescence microscopy. Overlay projections, representative of three assays, are shown. (C) Vimentin wt or C328S at $5 \mu \mathrm{M}$ in hypotonic buffer $(5 \mathrm{mM}$ PIPES-Na pH 7.0, 0.2 mM DTT) was deposited on a glass coverslip, and the control image was obtained immediately thereafter. Subsequently, $\mathrm{ZnCl}_{2}$ or $\mathrm{MgCl}_{2}$ was added at the indicated final concentrations, and bright field images were obtained within $10 \mathrm{~min}$ of addition at r.t. Finally, zinquin was added at $0.6 \mathrm{mM}$, final concentration, and structures were visualized by UV detection. Results shown are representative of at least three independent assays with similar results. Scale bars, $20 \mu \mathrm{m}$. 
A
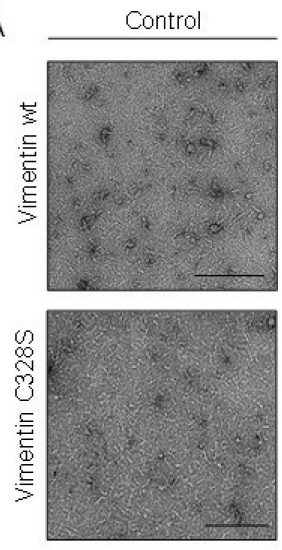

B

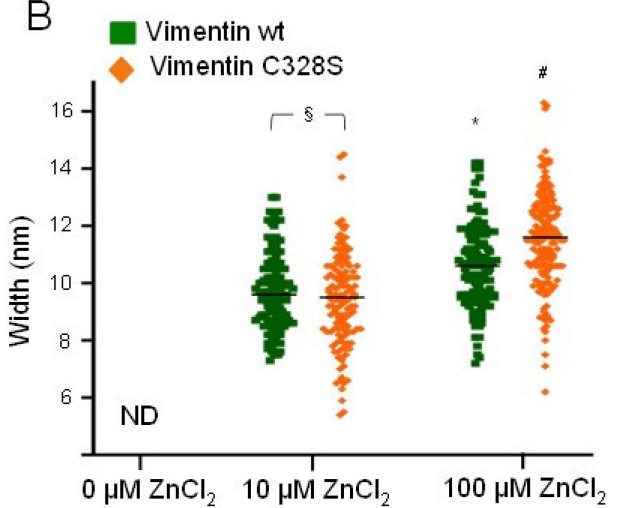

$10 \mu \mathrm{M} \mathrm{ZnCl}_{2}$
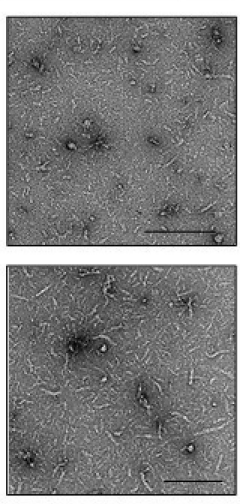
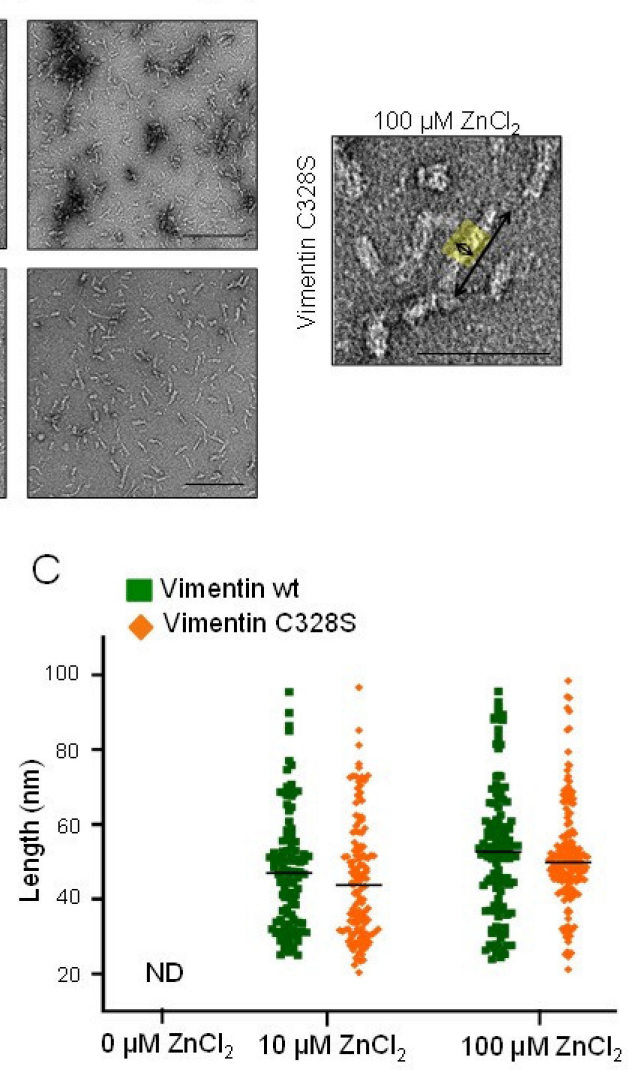

Figure 5. Observation of zinc-induced vimentin structures by electron microscopy. (A) Vimentin wt or C328S was incubated with the indicated concentrations of $\mathrm{ZnCl}_{2}$ for $1 \mathrm{~h}$ at r.t., fixed, negatively stained, and inspected by EM. Scale bars, $200 \mathrm{~nm}$ and $100 \mathrm{~nm}$ for the right panel. The width and length of the observed fibrils were measured using FIJI software as illustrated in the right panel, and results are summarized in the graphs. (B) Width of the structures of at least $20 \mathrm{~nm}$ in length. (C) Length of the structures observed. Results shown are the mean values \pm SEM of 50 "structures" per condition. $\S p<0.05$ vimentin wt vs. C328S, as indicated; ${ }^{*} p<0.05$ vs. vimentin wt with $10 \mu \mathrm{M} \mathrm{ZnCl}_{2} ; \# p<0.05$ vs. vimentin C328S with $10 \mu \mathrm{M} \mathrm{ZnCl} 2$ by the unpaired Student's $t$-test.

\subsection{Effect of Zinc on Nacl-Induced Polymerization and on Preformed Filaments}

Vimentin polymerization in cells occurs in an isotonic medium, which, in vitro, is usually mimicked by adding millimolar concentrations of monovalent salts, i.e., $\mathrm{NaCl}$ or $\mathrm{KCl}$. Therefore, turbidity assays were used to explore the effect of sequential addition of $\mathrm{NaCl}$ and $\mathrm{ZnCl}_{2}$ in different orders (Figure 6A). The addition of $150 \mu \mathrm{M} \mathrm{ZnCl}_{2}$ to vimentin rapidly increased turbidity. Subsequent addition of $150 \mathrm{mM} \mathrm{NaCl}$ caused a marked and immediate increase in turbidity, suggesting the formation of larger assemblies (Figure 6A, left panel). Conversely, the addition of $\mathrm{ZnCl}_{2}$ to vimentin after incubation with $\mathrm{NaCl}$ induced a more moderate increase in turbidity (Figure 6A, right panel). Then, the nature of the assemblies formed was assessed by electron microscopy. Strikingly, preincubation of vimentin with zinc precluded the formation of filaments upon the addition of $\mathrm{NaCl}$ and led to irregular assemblies that sometimes tended to form longitudinal arrays, and others appeared as tangled, non-linear structures (Figure 6B). This may indicate that the fibrils formed in the presence of zinc alone are not valid "starter units"; that is, they do not possess the proper alignment or conformation to undergo end-to-end annealing and compaction. It is noteworthy that the disrupting effect of zinc on subsequent $\mathrm{NaCl}$-elicited elongation occurred with concentrations as low as $10 \mu \mathrm{M} \mathrm{ZnCl}_{2}$; that is, at a 2:1 zinc:vimentin ratio. 

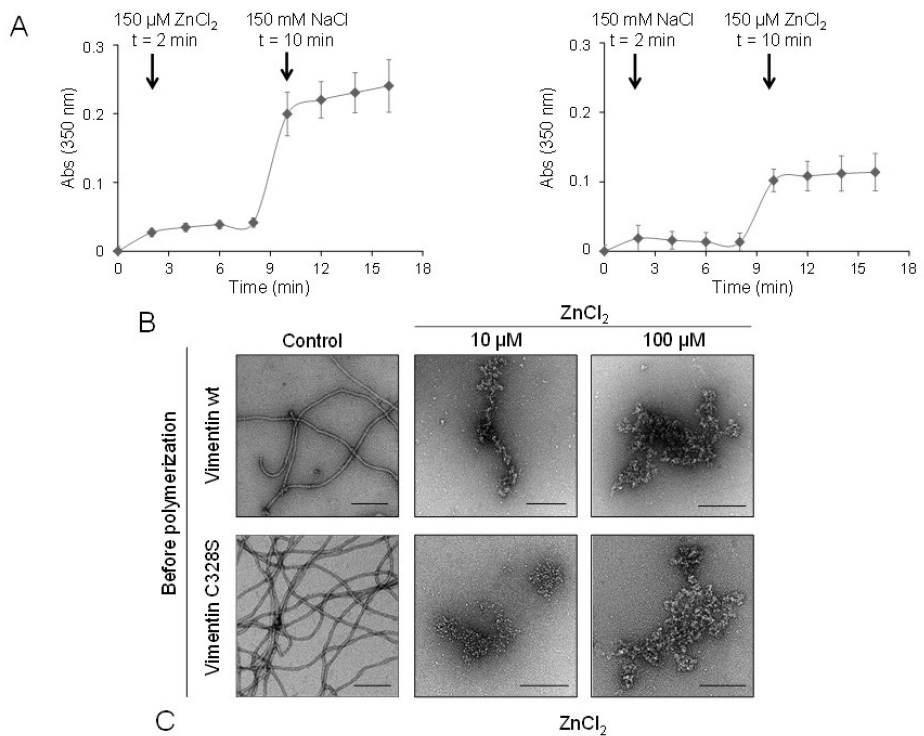

C
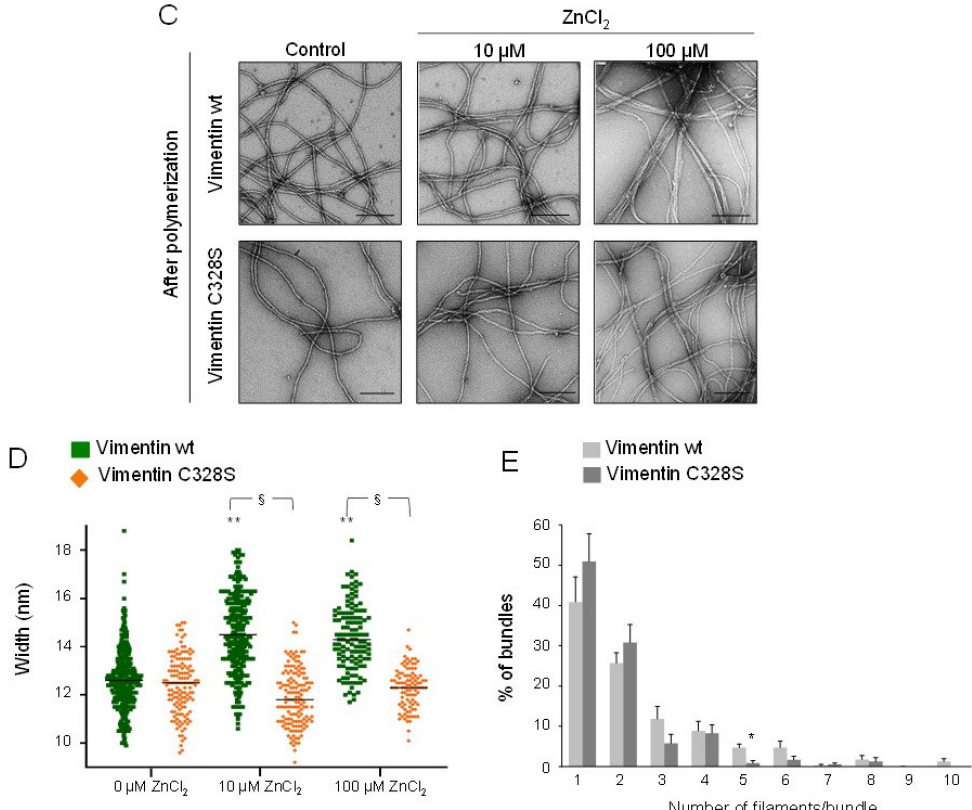

Figure 6. Impact of zinc on the assembly of soluble vimentin and on the morphology of preformed filaments. (A) The effect of $\mathrm{ZnCl}_{2}$ and $\mathrm{NaCl}$ added in different orders, as indicted, on the turbidity of vimentin solutions was monitored at r.t. Results are the average values \pm SD of three assays with similar results. (B) Effect of the preincubation of vimentin wt or $\mathrm{C} 328 \mathrm{~S}$ with vehicle or $\mathrm{ZnCl}_{2}$ at the indicated concentrations for $1 \mathrm{~h}$ at r.t. on subsequent polymerization by the addition of $150 \mathrm{mM} \mathrm{NaCl}$ and incubation for an additional hour at $37^{\circ} \mathrm{C}$. (C) Vimentin wt or C328S was first polymerized by incubation with $150 \mathrm{mM} \mathrm{NaCl}$ for $1 \mathrm{~h}$ at $37^{\circ} \mathrm{C}$, after which, $\mathrm{ZnCl}_{2}$ at the indicated final concentrations was added, and incubation was continued for $1 \mathrm{~h}$ at r.t. The morphology of vimentin wt or C328S filaments was assessed by EM. The images shown are representative of at least three independent assays with similar results. Scale bars, $200 \mathrm{~nm}$. (D) The width of filaments shown in (C) was measured with FIJI and represented as a scatter plot. Results are the mean values \pm SEM of 50 filaments per experimental condition (** $p<0.01$ vs. wt-control; $\S p<0.05$ vimentin wt vs. C328S by Student's $t$-test). (E) Images from preformed filaments of vimentin wt or C328S treated with $100 \mu \mathrm{M} \mathrm{ZnCl}_{2}$ were inspected for filaments appearing as individual entities or forming laterally associated groups or bundles. Then, the percentage of bundles containing the indicated number of filaments was calculated. At least 230 bundles were counted per experimental condition. Results are the average values \pm SEM of four and three assays for vimentin wt and C328S, respectively ( ${ }^{*} p<0.02 \mathrm{vs}$. wt by Student's $t$-test). 
In contrast, when $\mathrm{NaCl}$-induced preformed vimentin filaments were incubated in the presence of zinc, filament integrity was not altered (Figure 6C). Nevertheless, a clear increase in filament width, from 12.6 to $14.3 \mathrm{~nm}$, was observed with the lowest $\mathrm{ZnCl}_{2}$ concentration assayed $(10 \mu \mathrm{M})$. Higher concentrations of $\mathrm{ZnCl}_{2}$, up to $100 \mu \mathrm{M}$, did not induce further increases in filament diameter (Figure 6C). Importantly, this effect was observed only in vimentin wt filaments, since vimentin C328S filaments remained unaltered in the presence of zinc (quantitated in Figure 6D). Moreover, $100 \mu \mathrm{M} \mathrm{ZnCl}_{2}$ affected preformed filaments by inducing lateral association, preferentially in vimentin wt. Thus, in vimentin $w t$, bundles contained more filaments on average ( $3.4 \pm 1.8$ in wt vs. $2.8 \pm 1.4$ in C328S vimentin, average \pm SD of over 200 bundles, $p<0.0001$ ). Moreover, vimentin wt bundles were tightly packed, with a poor definition of individual filaments. In contrast, vimentin C328S filaments exposed to $100 \mu \mathrm{M}$ $\mathrm{ZnCl}_{2}$ most frequently associated laterally in groups of two or three filaments that preserved their individual contours (Figure 6C). The distribution of filaments in bundles in vimentin wt and C328S is represented in Figure 6E, showing that vimentin wt appears more frequently than vimentin C328S in groups of three or more filaments, whereas the mutant appears more frequently than the $\mathrm{wt}$ in groups of one or two filaments.

\section{Discussion}

The regulation of vimentin filament assembly is not completely understood. Here, we showed that micromolar zinc exerted unique effects on the assembly of vimentin in vitro. Zinc induced the oligomerization of soluble vimentin into fibril-like structures apparently not competent for filament formation. However, in preformed filaments, it induced morphological changes consisting of filament widening and lateral association. These effects could have physiological and pathophysiological implications, as discussed below.

Importantly, the effects of zinc occurred at micromolar concentrations. Therefore, their characterization required minimizing the presence of metal chelators. Indeed, metal chelators such as EDTA or EGTA were previously shown to affect vimentin polymerization, reportedly by altering the ionic strength of the solution [42]. In addition, EDTA could interfere with vimentin assembly through other mechanisms, such as the occurrence of $\mathrm{pH}$ changes in solutions containing EDTA upon the addition of divalent cations [45]. Therefore, throughout this study, we used a virtually EDTA-free vimentin preparation [41].

In previous studies, we observed that zinc protected the vimentin single cysteine residue, C328, from alkylation [3], which has been proposed to be an indication of zinc binding [46]. Therefore, here, we explored the effect of zinc on both vimentin wt and a cysteine to serine mutant, C328S. The substitution of a cysteine residue by serine is considered the most conservative one regarding size and atomic composition. Although the single cysteine residue appears not to be essential for in vitro vimentin assembly [17], here, we observed subtle differences between the polymerization behavior of vimentin wt and C328S in vitro, consistent with a faster elongation and formation of narrower filaments in the case of the mutant. Interestingly, a vimentin C $328 \mathrm{~S}$ mutant has proven to be less efficient than vimentin wt for initial network formation and undergoes faster dynamics in cells [3], indicating that, functionally, serine cannot fully substitute for cysteine in vimentin. These observations, together with the evidence of the impact of cysteine oxidative modifications in filament formation [4], could imply that C328 is located at a position important for filament assembly.

Zinc-induced vimentin oligomerization is fully reversible, as suggested by previous observations [3], and demonstrated here by real-time monitoring of light scattering. The dimensions of zinc-induced vimentin fibrils appear to be different from those of tetramers or typical ULF. This could be due to a higher degree of compaction of tetramers or to an altered alignment of dimers or tetramers, not appropriate for ULF formation and elongation. Notably, the effect of zinc on the aggregation of soluble vimentin appears to be similar in the wt and in the C328S mutant. In this context, it should be taken into account that, although histidine and cysteine residues are the preferred ligands, serine residues can also participate in interactions with zinc [30]. Moreover, carboxylic amino acids, glutamate 
and aspartate, also provide sites for zinc interaction [35]. Thus, the presence of numerous carboxylic amino acids in the vimentin sequence could explain the ability of zinc to promote the aggregation of both wt and C328S mutant vimentin.

Remarkably, concentrations of zinc in the hundreds of micromolar or low millimolar range induce the appearance of optically detectable vimentin aggregates and fibers. This could be related to the polyelectrolyte properties of vimentin, as multivalent counterions, including $\mathrm{Ca}^{2+}, \mathrm{Mg}^{2+}$, and $\mathrm{Mn}^{2+}$ at concentrations of $10 \mathrm{mM}$, have been shown to induce bundling of proteins with negative charge densities at their surfaces, including actin and vimentin [18]. Moreover, counterions can induce the formation of polyelectrolyte protein lattices through crosslinking [18].

However, we observed that the effects of zinc on vimentin oligomerization could not be mimicked by similar concentrations of magnesium, highlighting the differences in the interaction of the two cations with vimentin. On the one hand, the concentrations of magnesium required to induce vimentin oligomerization, as monitored by light scattering, are approximately 50-fold higher than those of zinc. On the other hand, whereas zinc-induced oligomers appear to be elongation-incompetent units, magnesium has been shown to induce ULF and short filaments on its own [20,23]. Moreover, zinc-induced vimentin oligomerization precludes subsequent filament formation upon the addition of $\mathrm{NaCl}$, of both wt and C328S vimentin. In contrast, filaments formed by millimolar magnesium are potentiated by the addition of $\mathrm{NaCl}$ [20]. However, it has been also found that magnesium or other divalent cations can delay the elongation step of vimentin polymerization, likely through the interaction of numerous magnesium ions with the region of the tail, impeding the head-to-tail interaction necessary for elongation. In addition, magnesium could promote intertetrameric interactions that could slow down the elongation process [20]. Thus, the possibility exists that zinc induces crosslinking at other sites of the protein with different consequences for vimentin assembly.

Of note, under the conditions of the light scattering assay, micromolar $\mathrm{ZnCl}_{2}$ and millimolar $\mathrm{MgCl}_{2}$, but not $\mathrm{NaCl}$, increased the turbidity of the vimentin solution. This could be due to the bigger size of aggregates induced by both divalent salts, which were the only ones optically detectable.

Importantly, the effect of zinc on vimentin assembly is strikingly different depending on the polymerization state of the protein (schematized in Figure 7). As stated above, zinc apparently induces an atypical oligomerization of wt and C328S soluble vimentin. Conversely, zinc does not disrupt preformed filaments, but alters their morphology in a manner dependent on the presence of the cysteine residue. Thus, at low micromolar concentrations, zinc selectively increases the diameter of vimentin wt, but not $\mathrm{C} 328 \mathrm{~S}$ vimentin filaments. Interestingly, we observed that some oxidants and electrophiles disrupt filament assembly by a mechanism dependent on the presence of the cysteine residue and lead to the increased diameter of preformed filaments, which does not occur in vimentin C328S [4]. Therefore, modulation of the environment of the cysteine residue, either by oxidation or hypothetically by zinc binding, could preclude the interactions needed for elongation or induce loosening of preformed filaments.

Our results showed that zinc also induced bundling of preformed vimentin filaments, which appeared more intense in vimentin wt than in C328S, thus indicating that the presence of the cysteine residue contributed to this effect. Recent in vitro observations indicate that zinc binding could alter the rheological properties of vimentin filaments [21], causing a stiffening of the network at low concentrations, but inducing bundle formation at higher concentrations, which resulted in a softening of the network. In this study, calcium exerted similar effects, although at higher concentrations [21].

Interestingly, vimentin appears to bind zinc with high capacity. In cells, fluorescent probes for protein-bound zinc light-up cytoskeletal structures colocalizing with vimentin bundles [3]. Indeed, given the concentration of negative charge surfaces on cytoskeletal proteins, we and others have proposed that these proteins could act as buffers for counterions [18,39] or as a zinc reservoir in the case of vimentin [3]. Notably, in the cellular milieu, millimolar concentrations of magnesium and other divalent cations are present, as well as many other potential cofactors. Nevertheless, vimentin seems to bind zinc with higher affinity than other divalent cations. We previously observed that 
preincubation with millimolar magnesium slowed down zinc binding to vimentin [3]. Therefore, the interplay between several cations in their interaction with vimentin could occur. Remarkably, examples of interplay between different cations in the binding and/or regulation of protein function can be found in tubulin, as detailed below, and in the case of certain phosphatases, like protein tyrosine phosphatase $1 \mathrm{~B}$, which is activated by magnesium and inhibited by zinc [34].

Zinc modulation of vimentin assembly could have both physiological and pathophysiological implications. Since the interaction of zinc with vimentin filaments results in the formation of bundles, this could contribute to its structural role. We have previously observed that zinc-deficient fibroblasts from a patient with a genetic alteration of zinc transport (acrodermatitis enteropathica) displayed vimentin filaments/bundles narrower than those of cells from a control subject, a condition that could be improved by zinc supplementation [3]. With respect to pathological aspects, sub-retinal pigment epithelial deposits associated with age-related macular degeneration contain both high concentrations of zinc $[47,48]$ and vimentin, among other proteins [49-51], which could lead to speculating about a role of zinc and vimentin in the formation or progression of these aggregates. Regarding pathological protein aggregates, micromolar zinc has been reported to induce aggregation of other proteins, including $A \beta$ and Tau in the context of neurodegenerative diseases $[31,52,53]$, factor $\mathrm{H}$ in relation to age-related macular degeneration [54], and tubulin, inducing atypical polymerization [55]. In the case of tubulin, zinc has been shown to induce the formation of sheets by mechanisms independent of the physiological polymerization elicited by GTP and magnesium [56]. However, a deficit in zinc availability can favor cysteine tubulin oxidation, causing cellular alterations [57], which reveals a complex interplay of zinc homeostasis and tubulin in cells. Analogously, zinc availability appears to modulate the susceptibility of the vimentin network to disruption by oxidants, which could occur by direct and indirect mechanisms [3].

\section{Materials and Methods}

Materials: Amicon ultrafiltration devices (10 K cut-off) were from Millipore. Slide-A-Lyzer MINI Dialysis devices (20 K cut-off) were from Thermo. PD-SpinTrap G-25 columns were from GE Healthcare. Ninety-six well clear plates were from Falcon. Carbon support grids, MESH CF 400 CU UL, used for electron microscopy were from Aname. High precision cells made of Quartz SUPRASIL, $10 \mathrm{~mm}$ path length, were purchased from Hellma. Sypro-Ruby for total protein staining was from Bio-Rad. The polar extract of E. coli phospholipids was from Avanti Polar Lipids. Human vimentin wt and C328S, expressed in E. coli and purified by established protocols [58], was obtained from Biomedal S.L. (Spain). Zinquin acid and N, N, N', N'tetrakis (2-pyridylmethyl) ethylenediamine (TPEN) were from Sigma. Alexa Fluor 488 carboxylic acid succinimidyl ester dye was from Molecular Probes, Invitrogen. High purity salts and other reagents were from Sigma and Merck.

Vimentin refolding and EDTA removal: This was achieved by ultrafiltration followed by dialysis, as previously described [41]. Briefly, vimentin preparations, in $8 \mathrm{M}$ urea, $5 \mathrm{mM}$ Tris- $\mathrm{HCl}, \mathrm{pH} 7.6$, $1 \mathrm{mM}$ EDTA, $10 \mathrm{mM} \beta$-mercaptoethanol, $0.4 \mathrm{mM} \mathrm{PMSF}$, and approximately $150 \mathrm{mM} \mathrm{KCl}$, were first diluted with EDTA-free buffer and ultrafiltrated using Millipore Amicon filter units (10 K pore size). Ultrafiltrated protein preparations were dialyzed step-wise against $5 \mathrm{mM}$ PIPES-Na, $\mathrm{pH}$ 7.0, $1 \mathrm{mM}$ DTT, containing decreasing urea concentrations $(6 \mathrm{M}, 4 \mathrm{M}, 2 \mathrm{M}$, and no urea), followed by two additional steps against $5 \mathrm{mM}$ PIPES-Na, pH 7.0, $0.25 \mathrm{mM}$ DTT, the last one for $16 \mathrm{~h}$ at $16^{\circ} \mathrm{C}$. Finally, proteins were centrifuged at $120,000 \times g$ for $15 \mathrm{~min}$ at $4{ }^{\circ} \mathrm{C}$, discarding the pellet, and aliquots were stored at $-80^{\circ} \mathrm{C}$. The protein concentration was estimated from its A280 nm, using an extinction coefficient of $22,450 \mathrm{M}^{-1} \mathrm{~cm}^{-1}$. The final DTT concentration in assays was kept below $0.2 \mathrm{mM}$ to minimize chelating effects [43]. 


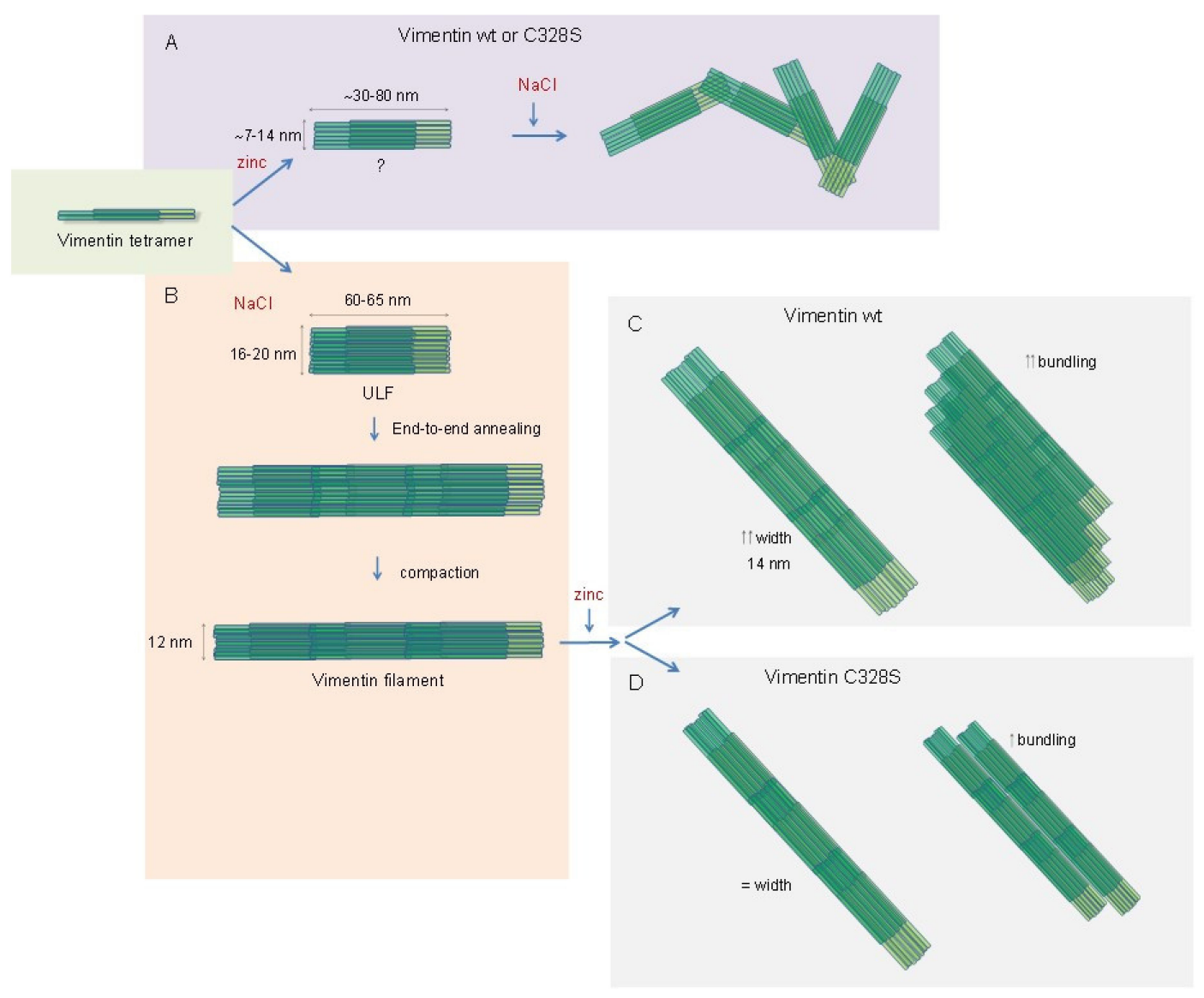

Figure 7. Scheme summarizing the impact of zinc on vimentin assembly as monitored by electron microscopy. Soluble vimentin, represented by a vimentin tetramer, forms atypical structures when incubated with $\mathrm{ZnCl}_{2}$ (A). Subsequent addition of $\mathrm{NaCl}$ does not produce filaments, but entangled aggregates. This effect is similar for vimentin wt and C328S. Conversely, incubation of soluble vimentin with $\mathrm{NaCl}$ (B) elicits the typical polymerization process, characterized by the formation of unit length filaments (ULF) and end-to-end annealing of these structures that are further compacted to form mature filaments. Incubation of these preformed filaments with zinc leads to a marked increase in width and intense bundling in the case of vimentin wt (C), whereas C328S mutant filaments do not suffer changes in width and undergo less intense bundling (D).

Polymerization assays: Vimentin wt and C328S, at $3.8 \mu \mathrm{M}$ in $5 \mathrm{mM}$ PIPES-Na, pH 7.0, 0.1 mM DTT, were incubated in the presence of $150 \mathrm{mM} \mathrm{NaCl}$ for $15 \mathrm{~min}$ at $37^{\circ} \mathrm{C}$ or with the indicated concentrations of $\mathrm{ZnCl}_{2}$ or $\mathrm{MgCl}_{2}$ for $1 \mathrm{~h}$ at room temperature (r.t.). In all situations, soluble (S, supernatant) and polymerized (P, pellet) fractions were separated by ultracentrifugation at $55,000 \mathrm{rpm}$ for $30 \mathrm{~min}$ at $4{ }^{\circ} \mathrm{C}$. Aliquots of both fractions were resuspended in Laemmli buffer and analyzed by SDS-PAGE, followed by total protein staining with Sypro-Ruby and ultraviolet (UV) light detection.

Turbidity assays: Solutions of vimentin wt and C328S, at $5 \mu \mathrm{M}$ in $5 \mathrm{mM}$ PIPES-Na, pH 7.0, were placed in Hellma absorption cuvettes, and increasing amounts of $\mathrm{ZnCl}_{2}, \mathrm{MgCl}_{2}$, or $\mathrm{NaCl}$ were sequentially added to achieve the indicated final concentrations. Turbidity of the incubation mixtures was immediately monitored at $350 \mathrm{~nm}$, at r.t., using an Agilent Cary $60 \mathrm{UV}$-Vis spectrophotometer. In the graphs, the absorbance values corresponding to the protein alone were subtracted and were corrected according to vimentin concentration and the volume of the mixtures. Time-dependent measurements were performed every $2 \mathrm{~min}$, for a total of $26 \mathrm{~min}$ in the analysis of the reversibility of the effect of $\mathrm{ZnCl}_{2}$ and for $16 \mathrm{~min}$ in the monitoring of the effect of $\mathrm{ZnCl}_{2}$ incubation before or after the addition of $\mathrm{NaCl}$. Turbidity of vimentin solutions without added salts and of incubation buffer 
with increasing concentrations of salts were monitored, and no changes in the turbidity were detected within the time frame of the experiments.

Protein labeling: Vimentin wt and C328S were covalently labeled in the amino groups by incubation with a three-fold molar excess of Alexa Fluor 488 carboxylic acid succinimidyl ester dye, after which, mixtures were subjected to gel filtration, using PD Minitrap G-25 columns, in order to eliminate the free dye. The extent of vimentin labeling was estimated from the molar absorption coefficients of $22,450 \mathrm{M}^{-1} \mathrm{~cm}^{-1}$ and of $71,000 \mathrm{M}^{-1} \mathrm{~cm}^{-1}$, at $280 \mathrm{~nm}$ for vimentin and at $495 \mathrm{~nm}$ for the dye, respectively, and ranged between 0.2 and 0.9 moles of fluorophore per mole of protein. Aliquots of vimentin were stored at $-80^{\circ} \mathrm{C}$, until assayed.

Fluorescence anisotropy: Mixtures of unlabeled vimentin $(1 \mu \mathrm{M})$ with a tracer amount of Alexa 488-labeled vimentin ( $50 \mathrm{nM}$ ) were incubated with different concentrations of $\mathrm{ZnCl}_{2}$, in a Corning 96 well black microplate. Fluorescence anisotropy was determined using a BMG POLARstar Galaxy plate reader, using $485 \mathrm{~nm}$ and $520 \mathrm{~nm}$ excitation and emission filters, respectively, at $26^{\circ} \mathrm{C}$.

Observation of vimentin assemblies induced by zinc on different supports: For the observation of vimentin assemblies formed in solution, $80 \mu \mathrm{L}$ aliquots of vimentin, at $5 \mu \mathrm{M}$ in $5 \mathrm{mM}$ PIPES-Na ( $\mathrm{pH}$ 7.0), $0.2 \mathrm{mM}$ DTT, were dispersed in a flat-bottomed microplate. Different concentrations of $\mathrm{ZnCl}_{2}$ were added to the protein solutions, and the structures formed after $20 \mathrm{~min}$ of incubation were visualized under a Zeiss optical inverted microscope coupled to an Axiocam ERc 5s camera.

For visualization of vimentin in microdroplets stabilized by lipids, Escherichia coli phospholipids dissolved in chloroform were dried under a nitrogen stream, and the resulting lipid film was resuspended in mineral oil to the final concentration by two cycles of vigorous vortexing and sonication in a water bath. Mixtures of unlabeled vimentin wt $(4 \mu \mathrm{M})$ and vimentin wt labeled with Alexa 488 $(1 \mu \mathrm{M})$ were incubated with vehicle or $\mathrm{ZnCl}_{2}$ at the indicated concentrations. Next, incubation mixtures were vigorously mixed with the $E$. coli lipids dispersed in mineral oil, and the resulting emulsions were applied on a glass coverslip and observed on a Leica SP2 confocal microscope.

For observation on glass slides, a $5 \mu \mathrm{L}$ aliquot of vimentin wt or C328S at $5 \mu \mathrm{M}$ was applied on a glass slide, and $5 \mu \mathrm{L}$ of vehicle, $200 \mu \mathrm{M}$, or $2.5 \mathrm{mM} \mathrm{ZnCl}_{2}$ or $\mathrm{MgCl}_{2}$ (final concentrations) were added and gently mixed with the pipette tip. Next, a coverslip was placed on top of the mixtures, and samples were visualized on a Leica SP5 confocal microscope using the bright field mode for image acquisition. For zinquin staining, zinquin acid was added to the mixtures at $0.6 \mathrm{mM}$ (final concentration). Fluorescence was detected by excitation with an ultraviolet laser at $405 \mathrm{~nm}$, and emission between 450 and $520 \mathrm{~nm}$ was acquired.

Electron microscopy: Soluble oligomeric species of vimentin at $0.2 \mathrm{mg} / \mathrm{mL}$ in $5 \mathrm{mM}$ PIPES-Na, pH 7.0 (hypotonic buffer), in the presence of $0.1 \mathrm{mM} \mathrm{DTT}$, were fixed with $0.1 \%(v / v)$ glutaraldehyde, final concentration. Vimentin filaments were obtained by inducing polymerization with $150 \mathrm{mM} \mathrm{NaCl}$, at $37^{\circ} \mathrm{C}$. Time-lapse monitoring of vimentin assembly was performed by fixing the mixtures at 5,10 , 60 , and $120 \mathrm{~min}$ after initiating the polymerization. The effect of divalent cations on vimentin assembly or preformed filaments was assessed by incubation with vehicle or the indicated concentrations of $\mathrm{ZnCl}_{2}$, for $1 \mathrm{~h}$ at r.t, before or after inducing vimentin polymerization by incubation with $150 \mathrm{mM}$ $\mathrm{NaCl}$, for $1 \mathrm{~h}$ at $37^{\circ} \mathrm{C}$. Experiments were carried out at least three times, and all incubations were performed in duplicate. Carbon support grids (MESH CF 400 CU UL, Aname) were laid onto drops of glutaraldehyde-fixed mixtures, subsequently washed with water, and negatively stained with $2 \%(w / v)$ uranyl acetate (Merck). Samples were observed in a JEOL JEM-1230 electron microscope operating at $100 \mathrm{kV}$, equipped with a CMOS TVIPS TemCam-F416 digital camera. EM images were obtained at $50 \mathrm{~K}$ magnification and were processed with FIJI software for the measurement of the width and length of vimentin oligomeric species and filaments. The "plot profile" plugin of straightened filaments was used to determine filament width. On average, 50 vimentin species were measured per experimental condition.

Statistical analysis: All assays were repeated at least three times. The results are presented as the mean values \pm standard error of the mean (SEM) or mean values \pm standard deviation (SD), as 
indicated. Statistical differences were evaluated by Student's t-test and were considered significant for $p<0.05$. The statistically significant differences are indicated on the graphs.

\section{Conclusions}

In summary, our observations indicate that zinc exerts complex effects on the organization of vimentin, depending on the polymerization state of the protein and on the presence of the cysteine residue. These differential effects could have implications in vimentin bundling or aggregation in physiological or pathophysiological conditions.

Author Contributions: A.M. performed the experiments, analyzed the data, prepared the illustrations, and wrote the manuscript; S.Z. designed the experiments, analyzed the data, and contributed to manuscript writing; G.R. provided feedback on the study design and interpretation of the results and critically read the manuscript; D.P.-S. designed and supervised the study and wrote the manuscript. All authors have read and agreed to the published version of the manuscript.

Funding: This work was supported by the European Union's Horizon 2020 research and innovation program under the Marie Sklodowska-Curie Grant Agreement No. 675132 “Masstrplan”, Grants SAF2015-68590-R and RTI2018-097624-B-I00 from Agencia Estatal de Investigación, MINECO/FEDER, Spain, and Instituto de Salud Carlos III/FEDER, RETIC Aradyal RD16/0006/0021. Feedback from COST Action CA15214 "EuroCellNet" is gratefully acknowledged.

Acknowledgments: We are indebted to Fernando Escolar and Rafael Núñez and Begoña Pou from CIB, CSIC, for valuable help with electron microscopy.

Conflicts of Interest: The authors declare no conflict of interest. The funders had no role in the design of the study; in the collection, analyses, or interpretation of data; in the writing of the manuscript; nor in the decision to publish the results.

\section{Abbreviations}

$\begin{array}{ll}\text { EM } & \text { Electron microscopy } \\ \text { TPEN } & \mathrm{N}, \mathrm{N}, \mathrm{N}^{\prime}, \mathrm{N}^{\prime} \text { tetrakis (2-pyridylmethyl) ethylenediamine } \\ \text { ULF } & \text { Unit length filament } \\ \text { wt } & \text { Wild-type }\end{array}$

\section{References}

1. Gan, Z.; Ding, L.; Burckhardt, C.J.; Lowery, J.; Zaritsky, A.; Sitterley, K.; Mota, A.; Costigliola, N.; Starker, C.G.; Voytas, D.F.; et al. Vimentin Intermediate Filaments Template Microtubule Networks to Enhance Persistence in Cell Polarity and Directed Migration. Cell Syst. 2016, 3, 252-263.e8. [CrossRef]

2. Duarte, S.; Viedma-Poyatos, Á.; Navarro-Carrasco, E.; Martínez, A.E.; Pajares, M.A.; Pérez-Sala, D. Vimentin filaments interact with the actin cortex in mitosis allowing normal cell division. Nat. Commun. 2019, 10, 4200. [CrossRef] [PubMed]

3. Pérez-Sala, D.; Oeste, C.L.; Martínez, A.E.; Jesús Carrasco, M.; Garzón, B.; Javier Cañada, F. Vimentin filament organization and stress sensing depend on its single cysteine residue and zinc binding. Nat. Commun. 2015, 6, 7287. [CrossRef] [PubMed]

4. Mónico, A.; Duarte, S.; Pajares, M.A.; Pérez-Sala, D. Vimentin disruption by lipoxidation and electrophiles: Role of the cysteine residue and filament dynamics. Redox Biol. 2019, 23, 101098. [CrossRef] [PubMed]

5. Antfolk, D.; Sjöqvist, M.; Cheng, F.; Isoniemi, K.; Duran, C.L.; Rivero-Muller, A.; Antila, C.; Niemi, R.; Landor, S.; Bouten, C.V.C.; et al. Selective regulation of Notch ligands during angiogenesis is mediated by vimentin. Proc. Natl. Acad. Sci. USA 2017, 114, E4574-E4581. [CrossRef] [PubMed]

6. Patteson, A.E.; Vahabikashi, A.; Pogoda, K.; Adam, S.A.; Mandal, K.; Kittisopikul, M.; Sivagurunathan, S.; Goldman, A.; Goldman, R.D.; Janmey, P.A. Vimentin protects cells against nuclear rupture and DNA damage during migration. J. Cell Biol. 2019, 218, 4079-4092. [CrossRef]

7. Hu, J.; Li, Y.; Hao, Y.; Zheng, T.; Gupta, S.K.; Parada, G.A.; Wu, H.; Lin, S.; Wang, S.; Zhao, X.; et al. High stretchability, strength, and toughness of living cells enabled by hyperelastic vimentin intermediate filaments. Proc. Natl. Acad. Sci. USA 2019, 116, 17175-17180. [CrossRef] 
8. Matsuyama, M.; Tanaka, H.; Inoko, A.; Goto, H.; Yonemura, S.; Kobori, K.; Hayashi, Y.; Kondo, E.; Itohara, S.; Izawa, I.; et al. Defect of mitotic vimentin phosphorylation causes microophthalmia and cataract via aneuploidy and senescence in lens epithelial cells. J. Biol. Chem. 2013, 288, 35626-35635. [CrossRef]

9. Battaglia, R.A.; Delic, S.; Herrmann, H.; Snider, N.T. Vimentin on the move: New developments in cell migration. F1000Research 2018, 7. [CrossRef]

10. Satelli, A.; Li, S. Vimentin in cancer and its potential as a molecular target for cancer therapy. Cell Mol. Life Sci. 2011, 68, 3033-3046. [CrossRef]

11. Mak, T.N.; Bruggemann, H. Vimentin in Bacterial Infections. Cells 2016, 5, 18. [CrossRef]

12. Musaelyan, A.; Lapin, S.; Nazarov, V.; Tkachenko, O.; Gilburd, B.; Mazing, A.; Mikhailova, L.; Shoenfeld, Y. Vimentin as antigenic target in autoimmunity: A comprehensive review. Autoimmun. Rev. 2018, 17, 926-934. [CrossRef]

13. Herrmann, H.; Aebi, U. Intermediate Filaments: Structure and Assembly. Cold Spring Harbor Perspect. Biol. 2016, 8, a018242. [CrossRef]

14. Premchandar, A.; Mücke, N.; Poznański, J.; Wedig, T.; Kaus-Drobek, M.; Herrmann, H.; Dadlez, M. Structural Dynamics of the Vimentin Coiled-coil Contact Regions Involved in Filament Assembly as Revealed by Hydrogen-Deuterium Exchange. J. Biol. Chem. 2016, 291, 24931-24950. [CrossRef] [PubMed]

15. Lopez, C.G.; Saldanha, O.; Aufderhorst-Roberts, A.; Martinez-Torres, C.; Kuijs, M.; Koenderink, G.H.; Köster, S.; Huber, K. Effect of ionic strength on the structure and elongational kinetics of vimentin filaments. Soft Matter 2018, 14, 8445-8454. [CrossRef] [PubMed]

16. Snider, N.T.; Omary, M.B. Post-translational modifications of intermediate filament proteins: Mechanisms and functions. Nat. Rev. Mol. Cell Biol. 2014, 15, 163-177. [CrossRef] [PubMed]

17. Rogers, K.R.; Herrmann, H.; Franke, W.W. Characterization of disulfide crosslink formation of human vimentin at the dimer, tetramer, and intermediate filament levels. J. Struct. Biol. 1996, 117, 55-69. [CrossRef]

18. Janmey, P.A.; Slochower, D.R.; Wang, Y.H.; Wen, Q.; Cēbers, A. Polyelectrolyte properties of filamentous biopolymers and their consequences in biological fluids. Soft Matter 2014, 10, 1439-1449. [CrossRef]

19. Lin, Y.C.; Broedersz, C.P.; Rowat, A.C.; Wedig, T.; Herrmann, H.; Mackintosh, F.C.; Weitz, D.A. Divalent cations crosslink vimentin intermediate filament tail domains to regulate network mechanics. J. Mol. Biol. 2010, 399, 637-644. [CrossRef]

20. Brennich, M.E.; Bauch, S.; Vainio, U.; Wedig, T.; Herrmann, H.; Köster, S. Impact of ion valency on the assembly of vimentin studied by quantitative small angle X-ray scattering. Soft Matter 2014, 10, 2059-2068. [CrossRef]

21. Wu, H.; Shen, Y.; Wang, D. Effect of the divalent cations zinc and calcium on the structure and mechanics of reconstituted vimentin intermediate filaments. BioRxiv 2019. [CrossRef]

22. Hofmann, I.; Herrmann, H.; Franke, W.W. Assembly and structure of calcium-induced thick vimentin filaments. Eur. J. Cell Biol. 1991, 56, 328-341. [PubMed]

23. Kooijman, M.; Bloemendal, M.; Traub, P.; van Grondelle, R.; van Amerongen, H. Transient electric birefringence study of intermediate filament formation from vimentin and glial fibrillary acidic protein. J. Biol. Chem. 1997, 272, 22548-22555. [CrossRef]

24. Brennich, M.E.; Vainio, U.; Wedig, T.; Bauch, S.; Herrmann, H.; Köster, S. Mutation-induced alterations of intra-filament subunit organization in vimentin filaments revealed by SAXS. Soft Matter 2019, 15, 1999-2008. [CrossRef] [PubMed]

25. Dammann, C.; Noding, B.; Koster, S. Vimentin networks at tunable ion-concentration in microfluidic drops. Biomicrofluidics 2012, 6, 22009-2200910. [CrossRef] [PubMed]

26. Dammann, C.; Koster, S. Dynamics of counterion-induced attraction between vimentin filaments followed in microfluidic drops. Lab Chip 2014, 14, 2681-2687. [CrossRef]

27. Kwak, H.I.; Kang, H.; Dave, J.M.; Mendoza, E.A.; Su, S.C.; Maxwell, S.A.; Bayless, K.J. Calpain-mediated vimentin cleavage occurs upstream of MT1-MMP membrane translocation to facilitate endothelial sprout initiation. Angiogenesis 2012, 15, 287-303. [CrossRef]

28. Inagaki, N.; Goto, H.; Ogawara, M.; Nishi, Y.; Ando, S.; Inagaki, M. Spatial patterns of Ca ${ }^{2+}$ signals define intracellular distribution of a signaling by $\mathrm{Ca}^{2+} /$ Calmodulin-dependent protein kinase II. J. Biol. Chem. 1997, 272, 25195-25199. [CrossRef] 
29. Heimfarth, L.; da Silva Ferreira, F.; Pierozan, P.; Loureiro, S.O.; Mingori, M.R.; Moreira, J.C.F.; da Rocha, J.B.T.; Pessoa-Pureur, R. Calcium signaling mechanisms disrupt the cytoskeleton of primary astrocytes and neurons exposed to diphenylditelluride. Biochim. Biophys. Acta 2016, 1860, 2510-2520. [CrossRef]

30. Maret, W. New perspectives of zinc coordination environments in proteins. J. Inorg. Biochem. 2012, 111, 110-116. [CrossRef]

31. Stefano, L.S.; Pierre, P.; Jae-Young, K.; Elias, A.; Ashley, I. Bush and Michal Hershfinkel. The neurophysiology and pathology of brain zinc. J. Neurosci. 2011, 31, 16076-16085.

32. Mo, Z.Y.; Zhu, Y.Z.; Zhu, H.L.; Fan, J.B.; Chen, J.; Liang, Y. Low micromolar zinc accelerates the fibrillization of human tau via bridging of Cys-291 and Cys-322. J. Biol. Chem. 2009, 284, 34648-34657. [CrossRef] [PubMed]

33. Cassandri, M.; Smirnov, A.; Novelli, F.; Pitolli, C.; Agostini, M.; Malewicz, M.; Melino, G.; Raschellà, G. Zinc-finger proteins in health and disease. Cell. Death Discov. 2017, 3, 17071. [CrossRef] [PubMed]

34. Bellomo, E.; Abro, A.; Hogstrand, C.; Maret, W.; Domene, C. Role of Zinc and Magnesium Ions in the Modulation of Phosphoryl Transfer in Protein Tyrosine Phosphatase 1B. J. Am. Chem. Soc. 2018, 140, 4446-4454. [CrossRef]

35. Maret, W. Zinc coordination environments in proteins as redox sensors and signal transducers. Antioxid Redox Signal. 2006, 8, 1419-1441. [CrossRef]

36. Maret, W. The redox biology of redox-inert zinc ions. Free Radic. Biol. Med. 2019, 134, 311-326. [CrossRef]

37. Fukuyama, K.; Murozuka, T.; Caldwell, R.; Epstein, W.L. Divalent cation stimulation of in vitro fibre assembly from epidermal keratin protein. J. Cell Sci. 1978, 33, 255-263.

38. Mack, J.W.; Steven, A.C.; Steinert, P.M. The mechanism of interaction of filaggrin with intermediate filaments. The ionic zipper hypothesis. J. Mol. Biol. 1993, 232, 50-66. [CrossRef]

39. Pérez-Sala, D.; Oeste, C.L.; Sánchez-Gómez, F.J. Vimentin gets a new glow from zinc. Oncotarget 2015, 6, 15742-15743.

40. Mónico, A.; Zorrilla, S.; Pérez-Sala, D. Characterization of vimentin-zinc interaction and its impact on the response to electrophilic and oxidative stress. Free Rad. Biol. Med. 2017, 108, S46. [CrossRef]

41. Mónico, A.; Martínez-Senra, E.; Cañada, F.J.; Zorrilla, S.; Pérez-Sala, D. Drawbacks of dialysis procedures for removal of EDTA. PLoS ONE 2017, 12, e0169843. [CrossRef] [PubMed]

42. Mücke, N.; Wedig, T.; Bürer, A.; Marekov, L.N.; Steinert, P.M.; Langowski, J.; Aebi, U.; Herrmann, H. Molecular and biophysical characterization of assembly-starter units of human vimentin. J. Mol. Biol. 2004, 340, 97-114. [CrossRef] [PubMed]

43. Krezel, A.; Maret, W. The biological inorganic chemistry of zinc ions. Arch Biochem Biophys 2016, 611, 3-19. [CrossRef] [PubMed]

44. Sokolova, A.V.; Kreplak, L.; Wedig, T.; Mücke, N.; Svergun, D.I.; Herrmann, H.; Aebi, U.; Strelkov, S.V. Monitoring intermediate filament assembly by small-angle x-ray scattering reveals the molecular architecture of assembly intermediates. Proc. Natl. Acad. Sci. USA 2006, 103, 16206-16211. [CrossRef]

45. Sharpe, J.C.; London, E. Inadvertent concentrating of EDTA by ion exchange chromatography: Avoiding artifacts that can interfere with protein purification. Anal. Biochem. 1997, 250, 124-125. [CrossRef]

46. Pace, N.J.; Weerapana, E. A competitive chemical-proteomic platform to identify zinc-binding cysteines. ACS Chem. Biol. 2014, 9, 258-265. [CrossRef]

47. Lengyel, I.; Flinn, J.M.; Peto, T.; Linkous, D.H.; Cano, K.; Bird, A.C.; Lanzirotti, A.; Frederickson, C.J.; van Kuijk, F.J. High concentration of zinc in sub-retinal pigment epithelial deposits. Exp. Eye Res. 2007, 84, 772-780. [CrossRef]

48. Flinn, J.M.; Kakalec, P.; Tappero, R.; Jones, B.; Lengyel, I. Correlations in distribution and concentration of calcium, copper and iron with zinc in isolated extracellular deposits associated with age-related macular degeneration. Met. Integr. Biometal Sci. 2014, 6, 1223-1228. [CrossRef]

49. Thompson, R.B.; Reffatto, V.; Bundy, J.G.; Kortvely, E.; Flinn, J.M.; Lanzirotti, A.; Jones, E.A.; McPhail, D.S.; Fearn, S.; Boldt, K.; et al. Identification of hydroxyapatite spherules provides new insight into subretinal pigment epithelial deposit formation in the aging eye. Proc. Natl. Acad. Sci. USA 2015, 112, 1565-1570. [CrossRef]

50. Booij, J.C.; Baas, D.C.; Beisekeeva, J.; Gorgels, T.G.; Bergen, A.A. The dynamic nature of Bruch's membrane. Prog. Retin. Eye Res. 2010, 29, 1-18. [CrossRef] 
51. Crabb, J.W.; Miyagi, M.; Gu, X.; Shadrach, K.; West, K.A.; Sakaguchi, H.; Kamei, M.; Hasan, A.; Yan, L.; Rayborn, M.E.; et al. Drusen proteome analysis: An approach to the etiology of age-related macular degeneration. Proc. Natl. Acad. Sci. USA 2002, 99, 14682-14687. [CrossRef] [PubMed]

52. Hu, J.Y.; Zhang, D.L.; Liu, X.L.; Li, X.S.; Cheng, X.Q.; Chen, J.; Du, H.N.; Liang, Y. Pathological concentration of zinc dramatically accelerates abnormal aggregation of full-length human Tau and thereby significantly increases Tau toxicity in neuronal cells. Biochim. Biophys Acta Mol. Basis Dis. 2017, 1863, 414-427. [CrossRef] [PubMed]

53. McCord, M.C.; Aizenman, E. The role of intracellular zinc release in aging, oxidative stress, and Alzheimer's disease. Front. Aging Neurosci. 2014, 6, 77. [CrossRef] [PubMed]

54. Nan, R.; Farabella, I.; Schumacher, F.F.; Miller, A.; Gor, J.; Martin, A.C.; Jones, D.T.; Lengyel, I.; Perkins, S.J. Zinc binding to the Tyr402 and His402 allotypes of complement factor H: Possible implications for age-related macular degeneration. J. Mol. Biol. 2011, 408, 714-735. [CrossRef] [PubMed]

55. Eagle, G.R.; Zombola, R.R.; Himes, R.H. Tubulin-zinc interactions: Binding and polymerization studies. Biochemistry 1983, 22, 221-228. [CrossRef]

56. Melki, R.; Carlier, M.F. Thermodynamics of tubulin polymerization into zinc sheets: Assembly is not regulated by GTP hydrolysis. Biochemistry 1993, 32, 3405-3413. [CrossRef]

57. Mackenzie, G.G.; Salvador, G.A.; Romero, C.; Keen, C.L.; Oteiza, P.I. A deficit in zinc availability can cause alterations in tubulin thiol redox status in cultured neurons and in the developing fetal rat brain. Free Radic. Biol. Med. 2011, 51, 480-489. [CrossRef]

58. Herrmann, H.; Hofmann, I.; Franke, W.W. Identification of a nonapeptide motif in the vimentin head domain involved in intermediate filament assembly. J. Mol. Biol. 1992, 223, 637-650. [CrossRef]

(C) 2020 by the authors. Licensee MDPI, Basel, Switzerland. This article is an open access article distributed under the terms and conditions of the Creative Commons Attribution (CC BY) license (http://creativecommons.org/licenses/by/4.0/). 